\title{
Muscular effects of statins in the elderly female: a review
}

\author{
This article was published in the following Dove Press journal: \\ Clinical Interventions in Aging \\ 17 January 2013 \\ Number of times this article has been viewed
}

\author{
Shilpa Bhardwaj',2 \\ Shalini Selvarajah ${ }^{3}$ \\ Eric B Schneider ${ }^{3}$ \\ 'Griffin Hospital, Derby, CT, USA; \\ ${ }^{2}$ Yale University, New Haven, CT, \\ USA; ${ }^{3}$ Center for Surgical Trials and \\ Outcomes Research, Johns Hopkins \\ School of Medicine, Baltimore, \\ MD, USA
}

Correspondence: Eric B Schneider Center for Surgical Trials and Outcomes Research, Department of Surgery, Johns Hopkins School of Medicine, 600 North Wolfe Street, Blalock 618, Baltimore, MD 21 287, USA

$\mathrm{Tel}+\mathrm{I} 4105022601$

Fax + I 4109558101

Email eschneil@jhmi.edu
Abstract: Statins have demonstrated substantial benefits in supporting cardiovascular health. Older individuals are more likely to experience the well-known muscle-related side effects of statins compared with younger individuals. Elderly females may be especially vulnerable to statin-related muscle disorder. This review will collate and discuss statinrelated muscular effects, examine their molecular and genetic basis, and how these apply specifically to elderly women. Developing strategies to reduce the incidence of statininduced myopathy in older adult women could contribute to a significant reduction in the overall incidence of statin-induced muscle disorder in this vulnerable group of patients. Reducing statin-related muscle disorder would likely improve overall patient compliance, thereby leading to an increase in improved short- and long-term outcomes associated with appropriate use of statins.

Keywords: statins, older adults, elderly, females, epidemiology

\section{Introduction}

Statins, or 3-hydroxy-3-methylglutaryl coenzyme A (HMG CoA) reductase inhibitors, represent a class of cholesterol-lowering drugs targeting low-density lipoproteins (LDLs). Nearly half of all men aged 65 years and above, and more than half of all women in the same age-group, meet diagnostic criteria for hyperlipidemia. ${ }^{1}$ Clinical trials involving prospective studies of individuals with hypercholesterolemia have demonstrated that long-term statin use is associated with a significant reduction in the risk of coronary artery disease (CAD) and other vascular disorders, including stroke. ${ }^{2}$ Based upon these findings, the primary and secondary prevention of CAD and stroke has led to statin drugs being widely prescribed to both male and female adults, often beginning in early middle age..$^{3-6}$

Since the introduction of lovastatin $\left(\right.$ Mevacor $^{\circledR}$, Merck and Co, Inc, Whitehouse Station, NJ, USA) to the US market in 1987, statins have become some of the most widely prescribed drugs both in the US and around the world. ${ }^{7}$ According to the IMS Institute for Healthcare Informatics review on the use of medicines in the US, in 2011 a total of 19.8 million Americans used cholesterol-lowering medicines regularly, spending approximately $\$ 20.1$ billion on these medications. Cholesterollowering medications as a group trail only oncologic and respiratory drugs in terms of annual patient pharmaceutical spending in the US. More than three-quarters of antihypercholesterolemia spending was for statins, with atorvastatin capturing more than half of all monies spent on statins. ${ }^{8}$ The proportion of statin use and 
spending associated specifically with women has not been well defined in current literature.

Statins are generally safe and effective, and represent a very useful intervention for the prevention of CAD and stroke. However, as is the case for any medication, adverse effects have been associated with the use of statins. One of the most common of these adverse effects is statin-induced myopathy. ${ }^{9-11}$ Statin-related muscle disorders constitute a clinical spectrum ranging from a generally painless increase in serum creatinine kinase $(\mathrm{CK})$ levels that do not exceed ten times the upper limit of normal (ULN), to a mild muscular discomfort termed myalgia, to potentially life-threatening rhabdomyolysis whereby myocytes degenerate, raising $\mathrm{CK}$ levels to beyond ten times the ULN, leading to renal dysfunction or failure in some individuals. ${ }^{9}$

Advancing age has been associated with increased risk of statin-induced muscle disorder across the entire spectrum, as well as with a significantly greater incidence of the more severe forms of this disorder reported among the oldest groups of statin users. ${ }^{9,12,13}$ To date, there have been relatively few reports on sex-specific relationships between advancing age and the incidence of myotoxic reactions among statin users. However, two published reports indicate that females demonstrate increased risk of developing muscle-related adverse events associated with statin use compared with men. ${ }^{3,9}$ In the relative vacuum of female-specific data on relationships between advancing age, statin use, and muscle dysfunction, this review will examine molecular and genetic factors associated with statin-induced myopathy, and how these factors specifically affect older adult women.

\section{Specific side effects on muscle systems Nomenclature}

There have been several different terminologies for the description of muscle-related adverse events associated with statin use. In order to better understand the nature of adverse events, the American College of Cardiology, American Heart Association, and National Heart, Lung, and Blood Institute (ACC/AHA/NHLBI) developed standards for classifying and reporting events across the spectrum of this disorder. ${ }^{9}$ The standardized descriptors range from myopathy, which is a nonspecific terminology representing any disease of the muscle, to myalgia, myositis, and at the most severe end of the spectrum, rhabdomyolysis, which can lead to acute renal failure and death. ${ }^{9}$ The National Lipid Association (NLA) and Food and Drug Administration (FDA), however, have different definitions for certain terms. ${ }^{10,14}$ We reproduce a table (Table 1) originally printed in a review by Joy and Hegele to illustrate the standardized definitions proposed by the ACC/AHA/NHLBI, compared to definitions employed by the NLA and FDA. ${ }^{14}$ Inconsistency in the ways in which these conditions are defined and described limits the ability to compare findings from one study to those of another because the adverse-event definitions used may differ markedly. ${ }^{7}$

\section{Incidence}

There is a great deal of variability in the reported incidence of statin-induced myopathy. The clinical advisory on the use and safety of statins, published in 2002 by Pasternak et al, cited an approximate $5 \%$ of study participants in clinical trials being affected by some form of statin-induced myopathy. ${ }^{9}$ Depending on myopathy definitions employed in clinical trials, the incidence can range from $0 \%$, such as was found in the Treating for New Targets (TNT) trial $(n=10,001)$, to $0.27 \%$, which was identified in the Scandinavian Simvastatin Survival Study $(4 \mathrm{~S})(\mathrm{n}=4444)$, and even up to $5 \%$ in other smaller trials. ${ }^{12,15,16}$ However, in observational studies, the incidence of myopathy among statin users was as high as $5 \%-10 \%{ }^{17,18}$

Rallidis et $\mathrm{al}^{6}$ enumerated several reasons that could explain this consistent underestimation of incidence rates in randomized clinical trials. These were primarily different definitions used to define myopathy, and the application of exclusion criteria that prevents patients with preexisting muscle symptoms or those at high risk for developing symptoms from being recruited into the trial. ${ }^{6}$ Additionally, the higher clinician-based incidence reports may be a function of extended clinician follow-up.

In the Collaborative Association Diabetes Study (CARDS), which evaluated the safety and tolerability of atorvastatin $10 \mathrm{mg}$ compared with placebo in 2838 diabetes patients aged 40-75 years with no history of coronary artery disease, the most common muscle symptoms reported were leg cramps and myalgia. However, the study reported fairly similar overall incidence of these disorders in both the treatment and placebo groups. ${ }^{19}$ Other studies have also shown that the incidence of statin-induce myopathy varies when it is administered as monotherapy instead of as combination therapy. The incidence is estimated to be $0.1 \%-0.5 \%$ with statin monotherapy and $0.5 \%-2.5 \%$ in combination therapies with other cholesterol-reducing drugs. ${ }^{20,21}$ Severe myopathy has been reported in $0.8 \%$ of patients on lovastatin and simvastatin, while fatal rhabdomyolysis rarely 
Table I Manifestations of myopathy according to ACC/AHA/NHLBI clinical advisory on the use of statins, NLA and FDA. Reprinted with permission Joy TR, Hegele RA. Narrative review: statin-related myopathy. ${ }^{14}$ @ Annals of Internal Medicine 2009

\begin{tabular}{|c|c|c|c|}
\hline \multirow[t]{2}{*}{ Term } & \multicolumn{3}{|l|}{ Definitions } \\
\hline & ACCIAHA/NHLBI & NLA & FDA \\
\hline Myopathy & Any disease of muscle & $\begin{array}{l}\text { Symptoms of myalgia (muscle pain or } \\
\text { soreness), weakness, or cramps, plus } \\
\text { creatine kinase }>10 \times \text { ULN }\end{array}$ & Creatine kinase $>10 \times$ ULN \\
\hline Myalgia & Muscle aches or weakness without CK elevation & NA & NA \\
\hline Myositis & Muscle symptoms with increased CK & NA & NA \\
\hline Rhabdomyolysis & $\begin{array}{l}\text { Muscle symptoms associated with marked } \\
\text { CK elevations, typically substantially over } \\
\text { ten times upper limit of normal }\end{array}$ & $\begin{array}{l}\text { Creatine kinase }>10,000 \mathrm{IU} / \mathrm{L} \text { or creatine } \\
\text { kinase }>10 \times \mathrm{ULN} \text { plus an elevation } \\
\text { in serum creatinine or medical intervention } \\
\text { with intravenous hydration }\end{array}$ & $\begin{array}{l}\text { Creatine kinase }>50 \times \text { ULN } \\
\text { and evidence of organ damage, } \\
\text { such as renal compromise }\end{array}$ \\
\hline
\end{tabular}

Abbreviations: ACC/AHA/NHLBI, American College of Cardiology/American Heart Association/National Heart, Lung, and Blood Institute; NLA, National Lipid Association; FDA, Food and Drug Administration; NA, not available; ULN, upper limit of normal; CK, creatine kinase.

occurs, with an incidence ranging between $0 \%$ and $0.1 \%$. A meta-analysis done recently using data available from 35 clinical trials did not find a significant difference in the incidence of rhabdomyolysis between statin treatment and placebo groups. ${ }^{22}$

Among elderly women, the specific subgroup of interest for this review, incidence rates for statin-related muscle disorders are rarely reported in the medical literature. However, in a trial with cerivastatin over a decade ago, a subgroup analysis on elderly women aged 65 years and above, whereby 90 subjects were assigned to $0.8 \mathrm{mg}$ cerivastatin and 27 subjects assigned to $0.4 \mathrm{mg}$ cerivastatin, revealed that myopathy incidence was $5.6 \%$ and $7.4 \%$, respectively. These incidence rates were higher than that seen in the overall study population, whereby incidence was consistently less than $2 \%$ in all study groups. ${ }^{23,24}$ In the medical review for FDA approval of the drug, elderly women $\geq 62$ years and weighing $\leq 65 \mathrm{~kg}$ had increased incidence of CK elevations more than ten times ULN, even though it was not considered significant enough at that time to disapprove the drug. ${ }^{24,25}$ Cerivastatin was eventually withdrawn from the market in the year 2001 after a ten- to 100-fold increased risk of mortality was observed among individuals using cerivastatin compared with those on other statin drugs. A lesson to be learned from this is that clinical trials typically do not have sufficient sample sizes, and they may not have adequate length of study to capture rarely occurring conditions, such as rhabdomyolysis. This is a concern for all statin trials that attempt to assess this potentially fatal component of statininduced myopathy.

A recent cross-sectional study performed on elderly women in Chile examined the association between statin use and loss of muscle mass and function. This small study compared 71 subjects on low-dose statins (rosuvastatin, lovastatin, simvastatin, or atorvastatin) with 57 subjects who were not on statins and had not taken a statin within the 2 months preceding the study. The investigators measured functional capacity by assessing quadriceps and hand-grip strength, and the time taken for subjects to perform the Timed Up and Go (TUG) test, as well as lean body mass and anthropometric measurements. They found no significant difference in the frequency of myalgia or in plasma CK levels between the two groups. They demonstrated that elderly women on low-dose statins did not appear to experience greater loss of muscle mass or function. On the contrary, in this study, statin users appeared to have better quadriceps strength and TUG time than noncurrent statin users; however, this association may have been confounded by participant socioeconomic status in that statin users tended to be from a higher socioeconomic background and may have benefited from unmeasured confounders, such as improved diet and greater physical activity. This study was limited by its small sample size, cross-sectional nature, and a study methodology that relied on self-reported behaviors (duration on statin treatment, muscle complaints, and level of physical activity). The results suggest that future prospective longitudinal studies employing objective methods for measuring muscle mass and function are needed to elucidate the relationship between statin use and temporal changes in muscle mass, strength, and functional ability. ${ }^{26}$

Current findings regarding sex-associated risks associated with statin use are more equivocal. A recent meta-analysis by Kostis et al did not detect any sex-specific differences in statin-related adverse effects. However, this study reported that women seemed to be underrepresented in statin clinical trials. $^{27}$ 


\section{Review of pharmacology and mode of action of statins related to effects on muscle \\ Competitive inhibitor of HMG CoA}

Statins are potent competitive inhibitors of HMG CoA, binding to HMG CoA reductase with three times more efficacy than the natural substrate. This inhibition leads to a disruption in the cholesterol biosynthesis pathway mediated by HMG CoA, thereby decreasing LDL cholesterol levels in the body. There is as yet no single, precise biochemical mechanism that has been implicated as the main cause of statin-induced myopathy. Several possible mechanisms have been discussed in the literature; however, the most common relates to statin's primary mechanism of action on the cholesterol biosynthetic pathway. ${ }^{28,29}$

\section{Reduced production of geranyl pyrophosphate and farnesyl pyrophosphate}

In vitro studies have demonstrated that statin-induced myopathy is most likely not due to the reduction in cholesterol synthesis itself. Instead, it is more likely due to the inhibition of the synthetic pathway, resulting in a reduction in the synthesis of crucial intermediary molecules such as geranyl pyrophosphate (GPP) and farnesyl pyrophosphate (FPP). GPP and FPP are responsible for the generation of various proteins essential in a variety of cellular signaling, transportation, and transformation processes that enhance cell-membrane integrity and support intracellular metabolic pathways. ${ }^{30,31}$

Apart from being intermediaries in the synthesis of cholesterol, GPP and FPP are also important in the prenylation, or posttranslational modification, of various cellular complexes, including proteins called lamins. Lamins are important for the structural and functional integrity of nuclei in cells by forming a nuclear lamina on the inner wall of the nucleus after interacting with nuclear membrane proteins. GPP and FPP are also precursors of central compounds, including dolichols and ubiquinone, which is also known as coenzyme $\mathrm{Q}_{10}\left(\mathrm{CoQ}_{10}\right)$. Dolichols help in glycosylation of intracellular polypeptides, a critical step in improving their function and thereby facilitating the formation of healthy structural proteins. $\mathrm{CoQ}_{10}$, on the other hand, is a hexameric compound found in the mitochondria of cells that plays a major role in the ultimate exchange of energy equivalents at the respiratory chain level. ${ }^{32}$

Statin-induced myopathy is hypothesized to occur via the following cascade: statin-induced disruption in the formation of GPP and FPP leads to dysprenylation of proteins, which drives production of dysfunctional lamins as well as dysfunctional Rab proteins and selenoproteins, which in turn interferes with transport of structural peptides within the cell, causing impaired intracellular signaling, sarcolemmal expression, structural alterations, and nuclear fragility. Concomitantly, the corresponding lack of dolichol and $\mathrm{CoQ}_{10}$ decreases expression of crucial membrane receptors responsible for cellular function and metabolism, leading to impaired energy production. Laboratory studies have shown that these changes tend to be more pronounced in skeletal muscle than in smooth muscle, which is why the cascade associated with the disruption of GPP and FPP has been thought to be an important driver of statin-induced myopathy. ${ }^{29}$

\section{$\mathrm{CoQ}_{10}$ depletion}

There have been consistent reports through animal as well as human studies on the correlation between statin administration and corresponding depletion of $\mathrm{CoQ}_{10}$. In humans particularly, there have been reports of a $20 \%-40 \%$ decrease in $\mathrm{CoQ}_{10}$ levels associated with various statin treatments. ${ }^{33}$ However, no direct association between decreased myocyte $\mathrm{CoQ}_{10}$ levels and myopathy has ever been demonstrated in any human or animal study. ${ }^{32,34}$ In fact, tissue levels of the $\mathrm{CoQ}_{10}$ enzyme have rarely been measured in any human study ${ }^{33}$ In addition, oral $\mathrm{CoQ}_{10}$ supplementation has not been shown to have any association with the risk of statin-related myopathy ${ }^{14}$ Furthermore, although this hypothesis implicating a disruption in the cholesterol synthesis pathway seems generally plausible, it does not explain why patients with hereditary hypocholesterolemia who are on statins do not report the occurrence of myopathy in the same way. ${ }^{35}$

\section{Myocyte-programmed cell death}

More recent studies have suggested that statins do affect cellular FFP levels, which can lead to a dose-dependent programmed cell-death phenomenon. This occurs when a decrease in FFP causes a decrease in prenylated forms of various Rab proteins. In addition to altering cellular mechanics, this process leads to an increase in intracellular calcium, which in turn activates caspase enzymes responsible for cell death. ${ }^{36}$ This mechanism of action may explain one aspect of the cholesterol-independent or pleiotropic effects of statins: therapeutic benefits may be related to statin-induced apoptosis in vascular smooth-muscle cells, which may lead to reduced atherosclerotic proliferation. However, in skeletal muscle cells, statin-induced apoptosis may lead to excessive cell mortality in susceptible individuals (perhaps, especially among older females) in whom myopathy ensues. ${ }^{37}$ 
Figure 1 illustrates key steps by which statin-induced musclecell damage is hypothesized to occur.

\section{Predisposing factors: sex, genetics, comorbidities, and drug interactions}

Aging causes changes in body composition and function, including reduction in hepatic and renal clearance that alters the pharmacodynamics and pharmacokinetics of drugs. ${ }^{38}$ The older one gets, the more likely one is to experience consequences of drug intensification leading to the manifestation of adverse effects, such as statin-induced myopathy. ${ }^{39,40}$

To complicate further the aging-related decline in bodily functions that alters drug effects, older adults are also more often inflicted with multiple comorbidities, some of which are likely to be treated pharmacologically, thereby exposing these individuals to the use of more drugs (polypharmacy). Both multiple comorbidities and polypharmacy worsen the already-compromised drug metabolic process in older aging individuals, leading to a heightened sensitivity to even low doses of drugs in general. ${ }^{38}$ As a class, statins are generally safe and well tolerated. However, the risk for developing statin-induced myopathy in the elderly is substantially higher compared to their younger, healthier counterparts. ${ }^{41}$

\section{Sex}

In general, adverse drug reactions are more common among females. ${ }^{9,42-45}$ It has been reported that females are 1.5-1.7 times more at risk for clinically relevant adverse drug reactions compared to males. ${ }^{44,45}$ In addition, a recent hospital-based study in Germany reported that elderly

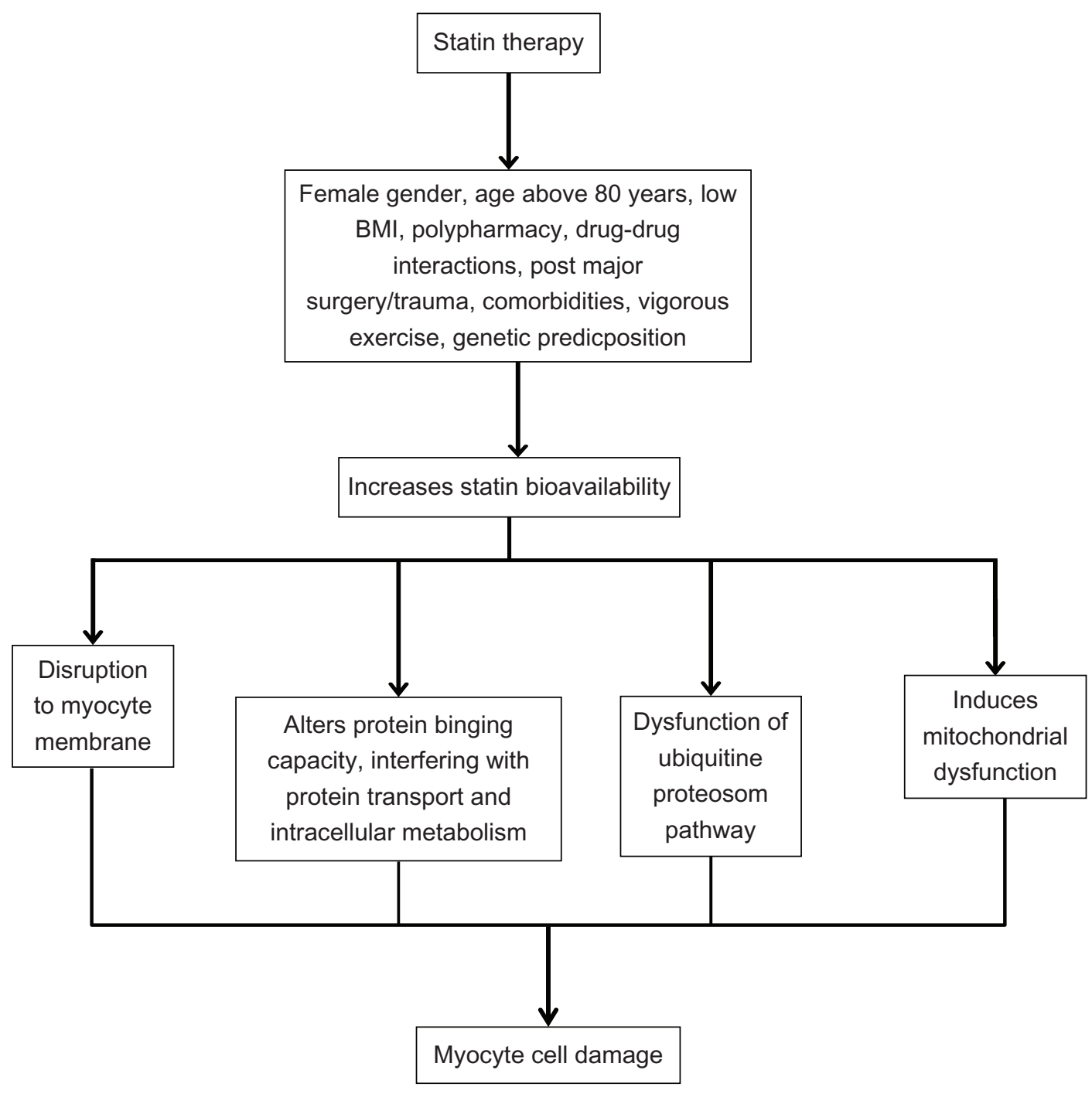

Figure I Conceptual diagram illustrating potential pathway by which statins induce myopathy. 
females had significantly higher rates of adverse drug-related hospital admissions compared to men. ${ }^{43}$ Evidence supporting sex-based differences in statin metabolism implicates, in part, well-known differences in body-fat content between men and women. This body fat-related differential drug metabolism is seen in other agents as well. ${ }^{42,46}$ This hypothesis has been well discussed in a general review on sex differences in pharmacological response that described the female preponderance towards adverse drug reactions. ${ }^{42}$ Although men weigh more than women in general, drug doses in elderly adults are rarely titrated based on weight. In some cases, this may expose frail, elderly women to a dose that is higher than their bodies are able to metabolize and eliminate efficiently. Females also tend to have a higher percentage of body fat, which affects volume of distribution of some drugs and can significantly increase the half-life of a variety of medications, including the more lipophilic statins. ${ }^{38,42,46}$

Most drugs are metabolized in the body through the hepatic cytochrome enzyme system. Lipophilic statins such as lovastatin, simvastatin, fluvastatin, atorvastatin, and pivastatin undergo first-pass metabolism in the liver, through reactions catalyzed by cytochrome P450 3A4 (CYP3A4). Women, however, have been found to have higher concentrations of CYP3A4. ${ }^{42,47,48}$ This should mean that females theoretically are more capable of clearing statins out of their bodies. Therefore, at first glance, it seems counterintuitive that females have a heightened susceptibility to adverse effects. However, as discussed above, other factors such as body weight and body fat may offset any differences in CYP3A4 concentrations. Also, polypharmacy is common among elderly women, and concomitant use of other drugs that are also metabolized by CYP3A4 may cause a drug-drug interaction related to the competitive need for CYP3A4. This competition may lead to lower than optimal clearance rates for one or more of the drugs, thereby increasing the possibility of adverse effects associated with higher than optimal drug levels. ${ }^{49,50}$

Significantly higher mean physiological levels of $\mathrm{CoQ}_{10}$ ( $1.11 \mathrm{vs} 0.86 \mu \mathrm{mol} / \mathrm{L}$ ) have been reported in males compared to females. ${ }^{51}$ Whether this significantly lower $\mathrm{CoQ}_{10}$ level in females predisposes them to myopathy compared with males has not been well documented in the literature. Also, other processes in the body that lead to $\mathrm{CoQ}_{10}$ depletion, such as diabetes and hypothyroidism, have been considered risk factors for statin-induced myopathy. ${ }^{6}$

It is also possible that a sociobehavioral perspective on pain perception may affect the likelihood of an individual complaining about discomfort or pain associated with statinrelated muscle disorder. ${ }^{52}$ Sex-based differences in pain perception favor a tendency for increased reporting of pain among females. There is a tendency for females to be more sensitive to pain and to describe pain as being more severe and recurrent in nature compared with males. ${ }^{53}$ A complex framework comprising biological, psychological, environmental, and sociological factors has been suggested to play a role in possible differences in pain reporting between males and females. ${ }^{52,53}$ Genetic influences on pain perception have also been significantly related to myalgia, but how genetically mediated pain perception is associated with greater statinrelated myopathies in elderly females is not well known. ${ }^{37}$

\section{Genetics}

Although statin-induced CK elevations are dose-dependent, the correlation between plasma levels of statins and the risk for statin-induced myopathy has not been consistently demonstrated across populations. ${ }^{37}$ To explain this discrepancy, there is a growing body of evidence describing various genetic factors that could contribute to differential reactions to the same drug from one individual to another. ${ }^{37}$

Some researchers have proposed that a synergistic interaction between genetic and pharmacologic nuances might be a possible mechanism of statin myopathy. ${ }^{54}$ It has been found that statin-induced myopathy is associated with a singlenucleotide polymorphism with intron 11 of the SLCO1B1 gene on chromosome $12 .{ }^{55}$ In the liver, statins enter hepatocytes using organic anion transport polypeptide (OATP) $1 \mathrm{~B} 1$, which is coded by the $S L C O 1 B 1$ gene. ${ }^{36,56}$ It has been reported that plasma statin concentration tends to be higher in people with the above polymorphism, thus predisposing them to adverse effects. ${ }^{55,57}$ However, recent studies have shown that this polymorphism might be significantly associated only with simvastatin-induced myopathy. ${ }^{58}$ Another recent population-based study showed that Native Americans might be at higher risk of having this polymorphism. ${ }^{59}$

Another multisite study identified three genes $-\mathrm{COQ}$, $A T P 2 B 1$, and $D M P K$ - that are responsible for pathways related to $\mathrm{CoQ}_{10}$ biosynthesis, calcium regulation in the body, and muscular dystonia, respectively, as markers for myalgia in patients having statin-associated myalgia. ${ }^{60}$ Benign CK elevation is also a marker for statin-induced myopathy. A retrospective case-control study involving 137 subjects taking simvastatin as a concomitant medication reported that nonexercise-induced $\mathrm{CK}$ elevation was associated with homozygosity in a genetic variant of the CYP3A enzyme, CYP3A $5 * 3$, which led to a greater degree of muscle damage. ${ }^{61}$ To the best of our knowledge, there is no literature reporting the distribution of these alleles based on sex. 


\section{Comorbidities}

Advanced age is a known risk factor for the presence of comorbid conditions. Without factoring in exposure to polypharmacy, comorbidities alone play a significant role in slowing statin metabolism and clearance from the body. Knowing that muscle-related effects of statins have been shown to increase in a dose-dependent manner, a health condition that could potentially lead to an accumulation of statins in plasma would be considered a risk factor for statininduced myopathy. ${ }^{36,62}$

Hepatic and renal insufficiencies are examples of conditions that would naturally increase the levels of statins circulating in plasma. ${ }^{11,39,63}$ Without titrating statin dose in patients suffering from such comorbid conditions, patients may face unnecessary overexposure to the drug when standard dosing regiments are applied. Dehydration is another risk factor for lower drug tolerance in the elderly. Though reduced water capacity may not be a diagnosis in itself, it is a condition that commonly accompanies other illnesses, especially in the elderly with limited self-care abilities. ${ }^{39}$ Dehydration can interfere with normal drug clearance by reducing the body's ability to eliminate drugs effectively, thus leading to higher than optimal drug levels. ${ }^{39}$ Figure 2 is an adaptation of a figure originally produced by Szadkowska et al, illustrating a combination of factors that could stem from or be exacerbated by the presence of comorbid conditions, each of which increases the overall risk of developing nonspecific drug-related adverse effects in older individuals. ${ }^{41}$

\section{Drug-drug interaction}

Negative drug-drug interactions involving statins occur when there is a drug-related disruption of the usual pharmacokinetic processes expected of statins. The dosedependent nature of statin-induced myopathy leads to the understanding that any concomitant use of drugs that could increase plasma concentration of statins increases the risk for developing this adverse effect. In addition,

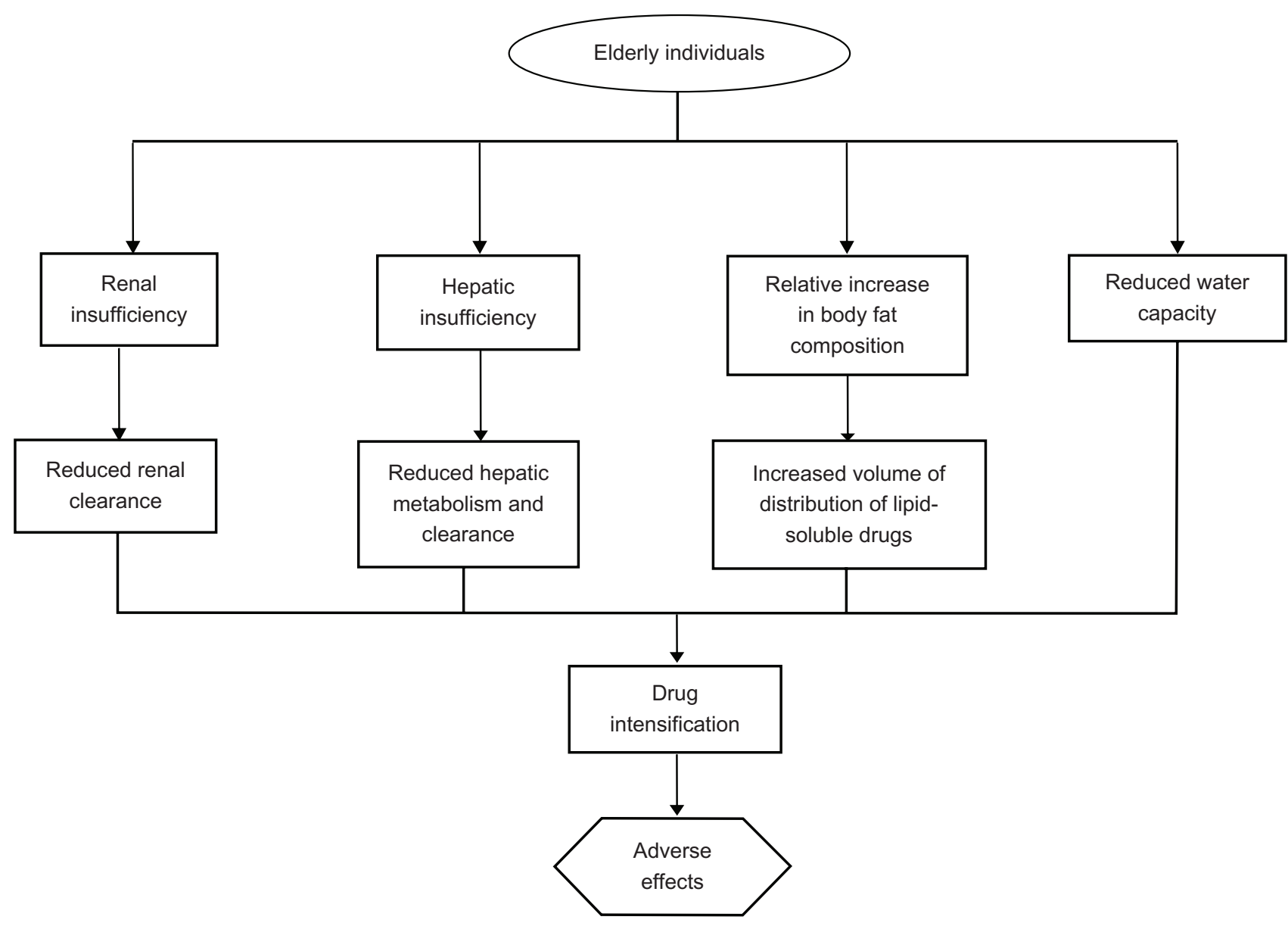

Figure 2 Major physical and physiologic factors adversely influencing statin pharmacokinetics in the elderly. 
agents that have the potential to alter statin pharmacodynamics, including statin response at the tissue level, also increase the likelihood for statin-induced myopathy to develop. ${ }^{39}$

Most concerns with statin-related drug-drug interactions that have been described in the literature are related to altered pharmacokinetic properties, whether at the level of drug absorption, distribution, metabolism, or excretion. Drug interaction-related increases in the bioavailability of statins can be a result of a number of different factors, including: increase in the uptake or absorption of statins from the gut; decreased hepatic blood flow limiting the amount of statin that is carried to the liver for first-pass metabolism; inhibition of renal excretion, especially in the case of renal insufficiency or reduced renal blood flow, which is an expected normal phenomenon in the elderly; and disrupted statin metabolism, especially if the other agents interact with the pathway by which the statin is normally metabolized. ${ }^{64}$

Drugs that utilize or interact with the CYP3A4 system tend to compete with predominantly lipophilic statins, such as simvastatin and atorvastatin, for CYP3A4. Drugs that are known to compete with lipophilic statins include amiodarone, azole antifungals, cyclosporine, calcium-channel blockers (eg, diltiazem), and antidepressants such as nefazodone, among others. ${ }^{65}$ Gemfibrozil, another cholesterol-lowering drug primarily targeting triglycerides, is a competitive inhibitor of certain cytochrome P450 isoenzymes (CYP) and uridine diphosphate glucuronyltransferase. Both of these enzymes are necessary for hepatic metabolism of certain statins. By inhibiting oxidation and glucuronidation processes, statin clearance is reduced, and this has been shown to increase plasma statin levels substantially. This inadvertently contributes to the higher risk of developing rhabdomyolysis, which has been reported among patients on statin-gemfibrozil combination therapy. ${ }^{66-68}$ Also, the inhibition of OATP1B1 by drugs such as cyclosporine and protease inhibitors (eg, ritonavir) increases plasma statin concentrations by reducing hepatic uptake of statins. ${ }^{69}$ A list of agents that have been described to influence statin bioavailability are presented in Table 2 .

\section{Other predisposing factors}

Of the seven statins presently used in practice, rosuvastatin and pravastatin are hydrophilic, while lovastatin, simvastatin, fluvastatin, atorvastatin, and pivastatin are more lipophilic. Hydrophilic statins are less capable of
Table 2 Agents implicated in increasing statin bioavailability and the associated mechanism of action

\begin{tabular}{ll}
\hline Agent & Mechanism of action \\
\hline Azole antifungals & Inhibits cytochrome P450 3A4 \\
Clarithromycin/erythromycin & \\
Diltiazem & \\
Verapamil & \\
Antiretroviral protease inhibitors & \\
Fibric acid derivatives & \\
Amiodarone & \\
Gemfibrozil & \\
Grapefruit juice & Competitively inhibits 3-hydroxy-3- \\
Red yeast rice & methylglutaryl coenzyme A \\
Cyclosporine & Inhibits organic anion-transporting \\
Phenytoin & polypeptide IBI transporter \\
Clopidogrel & Interferes with CYP29 metabolism \\
Sulphonamides & \\
\hline
\end{tabular}

entering nonhepatic cells. This is one possible reason why statin-induced myopathy appears to be reported less frequently with the use of rosuvastatin and pravastatin. However, it is important to note that this possible relationship between statin lipophilicity/hydrophylicity and muscle disorder remains a matter of debate. It is also argued that statin lipophilicity should be positively associated with the removal of statins from intracellular compartments. There is a possibility of physiochemical changes to statin molecules when entering cells, which then reduces their ability to be transported out of the cells; this possibility has not been entirely ruled out. Further study will be required to elucidate how different statins with different lipophilic properties and safety profiles are associated with the risk of myotoxicity. ${ }^{6,11}$

Other factors associated with statin-induced muscle dysfunction that have been mentioned in the literature include a history of muscle pain during previous statin treatments, previous unexplained muscle cramps, family history of muscle aches, and previous CK elevations. Increased physical activity has been implicated as a trigger of statin-induced myopathy. ${ }^{14,40,70}$ A 2011 study at the Boston Marathon found that statins appear to be associated with increased skeletal muscle injury, as evidenced by greater CK elevations in statin users $(n=37)$ compared to non-statin users $(n=40)$. However, the authors reported that instead of seeing a drug dose-dependent increase in risk, susceptibility to greater $\mathrm{CK}$ elevation appeared to be related to increasing age. ${ }^{70}$ Conditions that increase predisposition for statin-induced myopathy are listed in Table 3. 
Table 3 Risk factors for statin-induced myopathy

- Advanced age (particularly ages above 80 years)

- Female

- Small body frame and fragility (low body mass index)

- Multisystem disease (especially chronic renal insufficiency and diabetes)

- Untreated hypothyroidism

- Vigorous exercise

- Perioperative period (especially after major surgeries)

- Polypharmacy with risk for drug-drug interaction, especially with drugs that interfere with cytochrome P450 pathway

- Excessive alcohol intake

- Diet with excessive cranberry or grapefruit juice

- Genetic factors, ie, polymorphisms associated with cytochrome P450 isoenzymes, drug transporters, and myocyte metabolism

\section{Long-term safety and tolerability issues, and patient-focused perspectives such as quality of life}

The temporal relation between initiation of statin therapy and onset of myopathy remains unclear. Studies have reported symptom onset from anywhere between 1 and 12 months of initiating statin therapy. ${ }^{19} \mathrm{~A}$ small retrospective study involving 45 patients identified to have statin-induced myopathy between 1990 and 2003 reported a mean (standard deviation) duration of symptom onset since statin initiation as 6.3 (9.8) months. These patients also demonstrated a mean (standard deviation) duration for resolution of symptoms to be 2.3 (3.0) months after statins were discontinued. ${ }^{71}$ These studies, however, included patients of all ages, and to our knowledge, a temporal profile unique to the elderly, much less to elderly females, is not yet available. However, preliminary information relating a probable time course for the presentation of statin-induced myopathy supports the need to monitor symptoms and CK levels carefully, particularly in the first year, so that timely reassurance and intervention can be instituted where necessary.

Link et al also documented a twofold increase in risk for developing statin-induced myopathy in females after the first year of therapy, from a baseline first-year risk of 1.6, further supporting the agenda that greater care be taken with female patients being prescribed with statins. ${ }^{55}$ Again, it is difficult to ascertain, from this and other studies, a clear understanding of the interaction between age and sex in the development of statin-induced muscle disorder in older adults.

Among individuals suffering apparent statin-induced muscle disorder, muscular cramps and stiffness were the most commonly reported symptoms. ${ }^{10,72}$ Often, this was reported to be limited to the lower limbs. ${ }^{72}$ These symptoms were also considered endurable in most cases, but were reported as being severely incapacitating in some cases. ${ }^{10}$ In a survey done in 2003, Franc et al reported that approximately $90 \%$ of patients with muscle symptoms reported mild to moderate impact on daily activities, while $10 \%$ reported severe disability. More than a third of the patients in that study reported taking analgesics to ease their symptoms. ${ }^{72}$ In patients diagnosed with claudication, statin-induced myalgia was reported not to be severe enough to limit walking. ${ }^{73}$

Furthermore, most literature on statin-induced myopathy emphasizes that the disorder tends to be self-limiting and does not severely impact the daily functioning of afflicted statin users. Again, the current literature does not provide sufficient clarity to enable a complete understanding of ageand sex-related differences in the severity of patient-centered complaints regarding statin-induced muscle dysfunction. Patient-centered outcomes among statin users need to be elucidated more fully, and the limitations associated with patient complaints need to be studied in greater detail.

At present, there is a paucity of literature available to describe quality of life of patients with statin-induced myopathy, especially among elderly females. However, there is an ongoing study at Rockefeller University on quality of life among patients with statin-induced myopathy. To date, no interim findings have been reported; however, the results of this study should bring substantial knowledge regarding quality of life among statin users who suffer from statin-related muscle dysfunction. The results of this study should provide a solid foundation for future research in this important area, once they are made available.

Most studies that attempt to discuss quality of life among patients on statins have not focused exclusively on musclerelated complaints or changes, or on quality-of-life changes associated with the negative side effects of statins. Instead, these studies focused upon the improved quality and quantity of life enjoyed by statin users because of the successful prevention of adverse cardiovascular outcomes. ${ }^{41,74}$ Bearing in mind the substantial benefits associated with statin use, it is very important to recall that in general, drug-related adverse effects invariably result in reduced compliance. The occurrence of statin-induced myopathy compromises patient compliance in this way. ${ }^{41}$

Statin prescription, whether to achieve a therapeutic benefit or as a preventive measure, requires that there is consistent, prolonged use of the drug. Statin-induced myopathy becomes a major barrier for achieving these targets in patients who develop symptoms of muscular dysfunction. Fortunately, in light of the well-documented benefits of statin therapy, a variety of statin medications and doses are 
available to patients who have not tolerated one particular drug well. For example, there are statins that may have a better safety profile or that may be metabolized by a different metabolic pathway. Beyond statins, where indicated, other cholesterol-lowering drugs can be tried. It is interesting to note that statin rechallenge in a small study revealed equivocal findings, and further research is warranted into understanding which particular drug at which particular dose may enable any specific patient to enjoy the maximum benefit of statins with the least possible risk of encountering statin-related muscle dysfunction. ${ }^{69}$

Improving patient outcomes is central in medical care. Some aspects of care that physicians can keep in mind to increase safety and long-term tolerability of statins include the practice of prescribing statins at the lowest effective dose possible. Dose titration may be especially crucial in older female patients, given that elderly females have physiologic changes that predispose them to increased bioavailability of statins. Physicians should also strive to keep elderly females on as few medications as possible to avoid negative consequences of polypharmacy and minimize drug interactions with statins. When patients require drugs that may alter the metabolism of statins mediated by the CYP enzyme system, switching the patient to alternative statins, such as pravastatin and fluvastatin, which are hydrophilic and do not use the same metabolic pathway, should be considered in order to avoid drug-drug interactions and reduce the risk of possible statinrelated adverse effects. ${ }^{10,74}$ The ACC/AHA/NHLBI clinical advisory on statins recommends that patients hospitalized for major surgery with high metabolic demands might benefit from short-term cessation of statins in order to prevent statininduced myopathy in the perioperative period. ${ }^{9}$ However, there has been some concern about a possible "rebound effect" when statins are discontinued, and several small trials have demonstrated a reduction in perioperative morbidity and mortality among vascular surgery patients who were continued on statin therapy compared to those who had discontinued treatment prior to surgery. ${ }^{75-77}$ Sustained-release formulations, those which do not require daily dosing of statins, have also been shown to be associated with fewer muscle-related adverse effects. ${ }^{14}$ Considering the use of sustained-release formulations may be particularly important when addressing the issue of polypharmacy and impaired metabolism secondary to comorbidities in elderly females.

\section{Discussion and conclusion}

There is a paucity of sex-and age-specific information in the area of statin-induced myotoxicity. Hence, this review takes on an overall outlook of this topic, as reported by literature that is available.

Medication safety is a recognized indicator of quality of care. Measures to limit adverse drug reactions and medication-related adverse effects have been developed and implemented to varying degrees in most health-care settings. The incidence of statin-induced myopathy is relatively small, and this condition is usually self-limiting and relatively benign. However, because statins are so widely prescribed today, the experience with cerivastatin reminds us that the issue of medication safety in statins is vitally important.

Moving forward, it seems likely that increasing numbers of patients will be prescribed statins for more and more purposes. For example, there are studies being conducted to evaluate statin use in the management of conditions such as dementia, hypertension, and arthritis. ${ }^{69,77-80}$ The pleiotropic effect of statins extends their ability to offer cardioprotection to myocardial cells. A recent in vitro study revealed that pravastatin offered significant cardioprotection to isolated human myocardium exposed to hypoxic injury. In the vascular surgery arena, the use of statins prophylactically in the preoperative period is not uncommon. ${ }^{81}$ In addition, growing numbers of preclinical reports are revealing the potential for statins to enhance the effects of chemotherapeutic agents in cancer treatment, for varying types of cancers originating from different cell lines. ${ }^{82}$

Other recent reports include those suggesting the role of statins in the prevention of certain cancers (eg, hematopoietic cancers), as well as in improving recovery potential among patients with head injury. ${ }^{83,84}$ As the indications for statin use expand, more individuals will be exposed to the risk of statin-induced myopathy.

Of particular concern with statins is that long-term use is required in order for the statins to be effective in the treatment of chronic disorders such as hypercholesterolemia. Long-term use of statins is also required for the primary or secondary prevention of cardiovascular disease. Chronic exposure to statins not only increases one's likelihood of developing an adverse event just by virtue of continually challenging the body with the task of metabolizing the drug but also because of the long-term probability of developing other health conditions that may influence statin metabolism in a negative way. Currently, statin prescriptions are mostly concentrated among the middle-aged and the elderly. Even among statin users with good health, the aging process in itself inexorably increases the risk for statin-induced myopathy. The presence of greater comorbidity and exposure to polypharmacy in elderly individuals indicates that a careful focus on the 
safety of these patients is warranted when introducing any new medication, including a statin.

With this in mind, commencing statin therapy in elderly female patients, especially for primary or secondary prevention of cardiovascular disease, should follow a cautious approach. A thorough assessment of coronary mortality risk should be made, and only when clearly indicated should statins be prescribed, and then at an appropriately titrated dose. However, despite possible concerns about statin-induced muscle disorder, statin therapy should never be avoided in an appropriately screened patient when evidence shows the patient to be at high risk for coronary events. ${ }^{74,85}$ It is important to note that in the face of substantial level I evidence on the benefits of statins in primary and secondary prevention of coronary heart disease and stroke among the elderly, there still exists a significant therapeutic gap in this population, with a significant number of geriatric patients not reaching their therapeutic LDL goals. ${ }^{86}$

One reason behind the therapeutic gap seen in elderly statin users may be the failure of the physician to titrate the patient to an optimal dose, mainly for fear of statin-induced myopathy. A retrospective cohort study analyzed databases that included 396,077 elderly residents in Ontario, Canada with coronary artery disease and diabetes, and found that only $19 \%$ of patients were on statin therapy. In this particular study, patients prescribed statins were younger and more likely to be male. The authors suggested that physician misconceptions about the risk-benefit tradeoff of statins were responsible, at least in part, for the low rate of statin prescriptions among these at-risk patients. ${ }^{86}$

A recent nationwide review in the US revealed a reduction in spending for retail prescription of statins among the elderly. ${ }^{8}$ It is interesting to note that advanced age has been shown to be an independent risk factor for underutilization of statins..$^{41}$ This is not unusual, as compliance is a recognized issue if a patient experiences any form of adverse effect, or if patients are unable to obtain the medications, whether due to financial reasons that prohibit them from continuing to purchase the medication in a pharmacy, or because of the lack of independence to travel to a facility to retrieve these medications. Improving evidence on the influence of aging, sex, and other patient-specific data on statin-induced myopathy would assist physicians in making risk-benefit tradeoffs when prescribing statins in practice. In the future, it may be possible for physicians to use the genetic profiles of their patients to guide their choice to prescribe statin medications and guide the choice of drug and dosage. ${ }^{37,55,58}$
Despite the vast amount of information available in the field of statins and their adverse effects on muscles, the search for articles focusing only on elderly females was a futile effort. With the exception of the Chilean trial, none of the other cited studies focused specifically on the elderly female. Hence, this review strove to highlight essential findings that could be applied to elderly females based on existing knowledge on human physiology and the aging process. Further work is warranted to understand fully how statin-associated adverse effects actually affect elderly women.

\section{Limitations}

Recognizing potential risk factors for statin-induced muscle disorders is crucial. However, to properly develop strategies to address the increased risk of developing statin-induced myopathy in elderly women, the field requires further studies focusing on this population. The high variability in incidence of statin-induced myopathy reported in the literature could be attributed to the different definitions of myopathy employed in various trials, and the underrepresentation of women in some of them..$^{27,87}$ Although statins have demonstrated a good safety profile in clinical trials, it is not unreasonable to hypothesize that muscle-related adverse effects might be significantly higher in the unmonitored, sicker, and older populations. Statin-induced muscle effects remain a concern, despite being generally mild and self-limiting. The paucity of information related to elderly females calls for more research focusing on this vulnerable subpopulation.

Developing strategies to reduce the incidence of statininduced myopathy in older adult women could contribute to a significant reduction in the overall incidence of statininduced muscle disorder in this vulnerable group of patients. Reducing statin-related muscle disorder would be likely to improve overall patient compliance, thereby leading to an increase in the improved short- and long-term outcomes associated with appropriate use of statins.

\section{Disclosure}

The authors report no conflicts of interest in this work.

\section{References}

1. National Center for Health Statistics. Health, United States, 2010: With Special Feature on Death and Dying. Hyattsville (MD): National Center for Health Statistics; 2011.

2. Baigent C, Keech A, Kearney PM, et al. Efficacy and safety of cholesterol-lowering treatment: Prospective meta-analysis of data from 90,056 participants in 14 randomised trials of statins. Lancet. 2005;366: 1267-1278.

3. Walsh JME, Pignone M. Drug treatment for hyperlipidemia in women. JAMA. 2004;291:2243-2252. 
4. Heart Protection Study Collaborative Group. MRC/BHF heart protection study of cholesterol lowering with simvastatin in 20,536 high-risk individuals: a randomised placebo-controlled trial. Lancet. 2002;360: $7-22$.

5. Lemaitre RN, Furberg CD, Newman AB, et al. Time trends in the use of cholesterol-lowering agents in older adults: the cardiovascular health study. Arch Intern Med. 1998;158:1761-1768.

6. Rallidis LS, Fountoulaki K, Anastasiou-Nana M. Managing the underestimated risk of statin-associated myopathy. Int J Cardiol. 2012;159: 169-176.

7. Bays H. Statin safety: an overview and assessment of the data -2005 . Am J Cardiol. 2006;97:6C-26C.

8. IMS Institute for Health Informatics. The Use of Medicines in the United States: Review of 2011. Parsippany, NJ: IMS Institute for Health Informatics; 2012. Available from: http://www.imshealth.com/ims/ Global/Content/Insights/IMS $\% 20$ Institute $\% 20$ for $\% 20$ Healthcare $\% 20$ Informatics/IHII_Medicines_in_U.S_Report_2011.pdf. Accessed October 15, 2012.

9. Pasternak RC, Smith SC Jr, Bairey-Merz CN, et al. ACC/AHA/ NHLBI clinical advisory on the use and safety of statins. Circulation. 2002;106:1024-1028.

10. McKenney JM, Davidson MH, Jacobson TA, Guyton JR; National Lipid Association Statin Safety Assessment Task Force. Final conclusions and recommendations of the national lipid association statin safety assessment task force. Am J Cardiol. 2006;97:89C-94C.

11. Sathasivam S. Statin induced myotoxicity. Eur J Intern Med. 2012;23: 317-324.

12. Alexander KP, Blazing MA, Rosenson RS, et al. Management of hyperlipidemia in older adults. J Cardiovasc Pharmacol Ther. 2009;14: $49-58$.

13. Gaist D, Rodríguez LA, Huerta C, Hallas J, Sindrup SH. Lipid-lowering drugs and risk of myopathy: a population-based follow-up study. Epidemiology. 2001;12:565-569.

14. Joy TR, Hegele RA. Narrative review: statin-related myopathy. Ann Intern Med. 2009;150:858-868.

15. LaRosa JC, Grundy SM, Waters DD, et al. Intensive lipid lowering with atorvastatin in patients with stable coronary disease. $N$ Engl $J$ Med. 2005;7(352):1425-1435.

16. Thompson PD, Clarkson P, Karas RH. Statin-associated myopathy. JAMA. 2003;289:1681-1690.

17. Nichols GA, Koro CE. Does statin therapy initiation increase the risk for myopathy? An observational study of 32,225 diabetic and nondiabetic patients. Clin Ther. 2007;29:1761-1770.

18. Bruckert E, Hayem G, Dejager S, Yau C, Bégaud B. Mild to moderate muscular symptoms with high-dosage statin therapy in hyperlipidemic patients - the PRIMO study. Cardiovasc Drugs Ther. 2005;19: 403-414.

19. Newman CB, Szarek M, Colhoun HM, et al. The safety and tolerability of atorvastatin $10 \mathrm{mg}$ in the collaborative atorvastatin diabetes study (CARDS). Diab Vasc Dis Res. 2008;5:177-183.

20. Ballantyne CM, Corsini A, Davidson MH, et al. Risk for myopathy with statin therapy in high-risk patients. Arch Intern Med. 2003;163: 553-564.

21. Evans M, Rees A. Effects of HMG-CoA reductase inhibitors on skeletal muscle: Are all statins the same? Drug Saf. 2002;25:649-663.

22. Mills EJ, Wu P, Chong G, et al. Efficacy and safety of statin treatment for cardiovascular disease: a network meta-analysis of 170,255 patients from 76 randomized trials. QJM. 2011;104:109-124.

23. Insull W Jr, Isaacsohn J, Kwiterovich P, et al. Efficacy and safety of cerivastatin $0.8 \mathrm{mg}$ in patients with hypercholesterolaemia: the pivotal placebo-controlled clinical trial. Cerivastatin Study Group. J Int Med Res. 2008;28:47-68.

24. Jacobson TA. Statin safety: lessons from new drug applications for marketed statins. Am J Cardiol. 2006;97:44C-51C.

25. Center for Drug Evaluation and Research. Baycol medical review. 1999. Available from: http://www.accessdata.fda.gov/drugsatfda_docs/ nda/2000/20-740S008_Baycol_medr.pdf. Accessed October 10, 2012.
26. Boetje M, Bunout D, Barrera G, de la Maza MP, Leiva L, Hirsch S. Effects of statin use on functional capacity and muscle mass in elderly women. Ageing Res. 2011;2:35-39.

27. Kostis WJ, Cheng JQ, Dobrzynski JM, Cabrera J, Kostis JB. Metaanalysis of statin effects in women versus men. $J$ Am Coll Cardiol. 2012;59:572-582.

28. Kuncl RW. Agents and mechanisms of toxic myopathy. Curr Opin Neurol. 2009;22:506-515.

29. Vaklavas C, Chatzizisis YS, Ziakas A, Zamboulis C, Giannoglou GD. Molecular basis of statin-associated myopathy. Atherosclerosis. 2009; 202:18-28.

30. Nishimoto T, Tozawa R, Amano Y, Wada T, Imura Y, Sugiyama Y. Comparing myotoxic effects of squalene synthase inhibitor, T-91485, and 3-hydroxy-3-methylglutaryl coenzyme A (HMG-CoA) reductase inhibitors in human myocytes. Biochem Pharmacol. 2003;66:2133-2139.

31. Matzno S, Yamauchi T, Gohda M, et al. Inhibition of cholesterol biosynthesis by squalene epoxidase inhibitor avoids apoptotic cell death in L6 myoblasts. J Lipid Res. 1997;38:1639-1648.

32. Marcoff L, Thompson PD. The role of coenzyme Q10 in statinassociated myopathy: a systematic review. J Am Coll Cardiol. 2007;49: 2231-2237.

33. Bełtowski J, Wójcicka G, Jamroz-Wiśniewska A. Adverse effects of statins - mechanisms and consequences. Curr Drug Saf. 2009;4: 209-228.

34. Chatzizisis YS, Vaklavas C, Giannoglou GD. Coenzyme Q10 depletion: Etiopathogenic or predisposing factor in statin associated myopathy? Am J Cardiol. 2008;101:1071.

35. Baker SK. Molecular clues into the pathogenesis of statin-mediated muscle toxicity. Muscle Nerve. 2005;31:572-580.

36. Guijarro C, Blanco-Colio LM, Ortego M, et al. 3-hydroxy-3methylglutaryl coenzyme a reductase and isoprenylation inhibitors induce apoptosis of vascular smooth muscle cells in culture. Circ Res. 1998;83:490-500.

37. Ghatak A, Faheem O, Thompson PD. The genetics of statin-induced myopathy. Atherosclerosis. 2010;210:337-343.

38. Mangoni AA, Jackson SH. Age-related changes in pharmacokinetics and pharmacodynamics: basic principles and practical applications. $\mathrm{Br}$ J Clin Pharmacol. 2004;57:6-14.

39. ElDesoky ES. Pharmacokinetic-pharmacodynamic crisis in the elderly. Am J Ther.2007;14:488-498.

40. Parker BA, Thompson PD. Effect of statins on skeletal muscle: exercise, myopathy, and muscle outcomes. Exerc Sport Sci Rev. 2012;40: 188-194.

41. Szadkowska I, Stanczyk A, Aronow WS, et al. Statin therapy in the elderly: a review. Arch Gerontol Geriatr. 2010;50:114-118.

42. Anderson GD. Gender differences in pharmacological response. Int Rev Neurobiol. 2008;83:1-10.

43. Hofer-Dueckelmann C, Prinz E, Beindl W, et al. Adverse drug reactions (ADRs) associated with hospital admissions - elderly female patients are at highest risk. Int J Clin Pharmacol Ther. 2011;49:577-586.

44. Fattinger K, Roos M, Vergères $P$, et al. Epidemiology of drug exposure and adverse drug reactions in two Swiss departments of internal medicine. Br J Clin Pharmacol. 2000;49:158-167.

45. Tran C, Knowles SR, Liu BA, Shear NH. Gender differences in adverse drug reactions. J Clin Pharmacol. 1998;38:1003-1009.

46. Ciccone GK, Holdcroft A. Drugs and sex differences: a review of drugs relating to anaesthesia. Br J Anaesth. 1999;82:255-265.

47. Sica DA, Gehr TWB. Rhabdomyolysis and statin therapy: relevance to the elderly. Am J Geriatr Cardiol. 2002;11:48-55.

48. Wolbold R, Klein K, Burk O, et al. Sex is a major determinant of CYP3A4 expression in human liver. Hepatology. 2003;38:978-988.

49. Gruer PJ, Vega JM, Mercuri MF, Dobrinska MR, Tobert JA. Concomitant use of cytochrome P450 3A4 inhibitors and simvastatin. Am J Cardiol.1999;84:811-815.

50. Yoshida M, Matsumoto T, Suzuki T, Kitamura S, Mayama T. Effect of concomitant treatment with a CYP3A4 inhibitor and a calcium channel blocker. Pharmacoepidemiol Drug Saf. 2008;17:70-75. 
51. Kaikkonen J, Nyyssönen K, Tuomainen TP, Ristonmaa U, Salonen JT. Determinants of plasma coenzyme Q10 in humans. FEBS Lett. 1999; 443:163-166.

52. Racine M, Tousignant-Laflamme Y, Kloda LA, Dion D, Dupuis G, Choinière M. A systematic literature review of 10 years of research on sex/ gender and pain perception - part 2: do biopsychosocial factors alter pain sensitivity differently in women and men? Pain. 2012;153:619-635.

53. Goffaux P, Michaud K, Gaudreau J, Chalaye P, Rainville P, Marchand S. Sex differences in perceived pain are affected by an anxious brain. Pain 2011;152:2065-2073.

54. Vladutiu GD, Simmons Z, Isackson PJ, et al. Genetic risk factors associated with lipid-lowering drug-induced myopathies. Muscle Nerve. 2006;34:153-162.

55. Link E, Parish S, Armitage J, et al. SLCO1B1 variants and statininduced myopathy - a genome-wide study. N Engl J Med. 2008;359: 789-799.

56. Wilke RA, Ramsey LB, Johnson SG, et al. The clinical pharmacogenomics implementation consortium: CPIC guideline for SLCO1B1 and simvastatin-induced myopathy. Clin Pharmacol Ther. 2012;92:112-117.

57. Pasanen MK, Fredrikson H, Neuvonen PJ, Niemi M. Different effects of SLCO1B1 polymorphism on the pharmacokinetics of atorvastatin and rosuvastatin. Clin Pharmacol Ther. 2007;82:726-733.

58. Brunham LR, Lansberg PJ, Zhang L, et al. Differential effect of the rs4149056 variant in SLCO1B1 on myopathy associated with simvastatin and atorvastatin. Pharmacogenomics J. 2012;12:233-237.

59. Santos PC, Soares RA, Nascimento RM, et al. SLCO1B1 rs4149056 polymorphism associated with statin-induced myopathy is differently distributed according to ethnicity in the Brazilian general population: Amerindians as a high risk ethnic group. BMC Med Genet. 2011;12: 136.

60. Ruaño G, Windemuth A, Wu AH, et al. Mechanisms of statin-induced myalgia assessed by physiogenomic associations. Atherosclerosis. 2011;218:451-456.

61. Wilke RA, Moore JH, Burmester JK. Relative impact of CYP3A genotype and concomitant medication on the severity of atorvastatin-induced muscle damage. Pharmacogenet Genomics. 2005;15:415-421.

62. Thompson PD, Clarkson PM, Rosenson RS; National Lipid Association Statin Safety Task Force Muscle Safety Expert Panel. An assessment of statin safety by muscle experts. Am J Cardiol. 2006;97:69C-76C.

63. Blom DJ. Statin therapy for the octogenarian? J Endocrinol Metab Diabetes South Afr. 2012;17:37-42.

64. Bressler R, Bahl JJ. Principles of drug therapy for the elderly patient Mayo Clin Proc. 2003;78:1564-1577.

65. Arora R, Liebo M, Maldonado F. Statin-induced myopathy: the two faces of Janus. J Cardiovasc Pharmacol Ther. 2006;11:105-112.

66. Schneck DW, Birmingham BK, Zalikowski JA, et al. The effect of gemfibrozil on the pharmakonietics of rosuvastatin. Clin Pharmacol Ther. 2004;75:455-463.

67. Corsini A, Ceska R. Drug-drug interactions with statins: will pitavastatin overcome the statins' Achilles heel? Curr Med Res Opin. 2011;27: 1551-1562.

68. Jones P, Davidson M. Reporting rate of rhabdomyolysis with fenofibrate + statin versus gemfibrozil + any statin. Am J Cardiol. 2006;95:120-122.

69. Neuvonen PJ, Niemi M, Backman JT. Drug interactions with lipid lowering drugs: mechanisms and clinical relevance. Clin Pharmacol Ther. 2006;80:565-581.

Clinical Interventions in Aging

\section{Publish your work in this journal}

Clinical Interventions in Aging is an international, peer-reviewed journal focusing on evidence-based reports on the value or lack thereof of treatments intended to prevent or delay the onset of maladaptive correlates of aging in human beings. This journal is indexed on PubMed Central, MedLine, the American Chemical Society's 'Chemical Abstracts Ser-
70. Parker BA, Augeri AL, Capizzi JA, et al. Effect of statins on creatine kinase levels before and after a marathon run. Am J Cardiol. 2012;109: 282-287.

71. Hansen KE, Hildebrand JP, Ferguson EE, Stein JH. Outcomes in 45 patients with statin-associated myopathy. Arch Intern Med. 2005;165:2671-2676.

72. Franc S, Dejager S, Bruckert E, Chauvenet M, Giral P, Turpin G. A comprehensive description of muscle symptoms associated with lipid-lowering drugs. Cardiovasc Drugs Ther. 2003;17:459-465.

73. Mascitelli L, Pezzetta F. Does statin therapy interfere with the ability of claudicant patients to exercise? Vasc Endovascular Surg. 2007;41: 473.

74. Maroo BP, Lavie CJ, Milani RV. Efficacy and safety of intensive statin therapy in the elderly. Am J Geriatr Cardiol. 2008;17:92-100.

75. Kulik A, Ruel M. Statins and coronary artery bypass graft surgery: preoperative and postoperatice efficacy and safety. Expert Opin Drug Saf. 2009;8:559-571.

76. Desai $\mathrm{H}$, Aronow WS, Ahn C, et al. Incidence of perioperative myocardial infarction and of 2-year mortality in 577 elderly patients undergoing noncardiac vascular surgery treated with and without statins. Arch Gerontol Geriatr. 2010;51:149-151.

77. Jick H, Zornberg GL, Jick SS, Seshadri S, Drachman DA. Statins and the risk of dementia. Lancet. 2000;356:1627-1631.

78. Freeman DJ, Norrie J, Sattar N, et al. Pravastatin and the development of diabetes mellitus: evidence for a protective treatment effect in the West of Scotland Coronary Prevention Study. Circulation. 2001;103:357-362.

79. Spósito AC, Mansur AP, Coelho OR, Nicolau JC, Ramires JA. Additional reduction in blood pressure after cholesterol-lowering treatment by statins (lovastatin or pravastatin) in hypercholesterolemic patients using angiotensin-converting enzyme inhibitors (enalapril or lisinopril). Am J Cardiol. 1999;83:1497-1499.

80. Leung BP, Sattar N, Crilly A, et al. A novel anti-inflammatory role for simvastatin in inflammatory arthritis. J Immunol. 2003;170: $1524-1530$

81. Lemoine S, Allouche S, Coulbault L, et al. Mechanisms involved in cardioprotective effects of pravastatin administered during reoxygenation in human myocardium in vitro. Anesthesiology. 2012;116:824-833.

82. Osmak M. Statins and cancer: current and future prospects. Cancer Lett. 2012;324:1-12.

83. Lutski M, Shalev V, Porath A, Chodick G. Continuation with statin therapy and the risk of primary cancer: a population-based study. Prev Chronic Dis. 2012;9:E137.

84. Schneider EB, Efron DT, MacKenzie EJ, Rivara FP, Nathens AB, Jurkovich GJ. Premorbid statin use is associated with improved survival and functional outcomes in older head-injured individuals. J Trauma. 2011;71:815-819.

85. Maroo BP, Lavie CJ, Milani RV. Secondary prevention of coronary heart disease in elderly patients following myocardial infarction: are all HMG-CoA reductase inhibitors alike? Drugs Aging. 2008;25: 649-664.

86. Ko DT, Mamdani M, Alter DA. Lipid-lowering therapy with statins in high-risk elderly patients: the treatment-risk paradox. JAMA. 2004;291:1864-1870.

87. Sirvent P, Mercier J, Lacampagne A. New insights into mechanisms of statin-associated myotoxicity. Curr Opin Pharmacol. 2008;8: $333-338$.

\section{Dovepress}

vice' (CAS), Scopus and the Elsevier Bibliographic databases. The manuscript management system is completely online and includes a very quick and fair peer-review system, which is all easy to use. Visi http://www.dovepress.com/testimonials.php to read real quotes from published authors. 